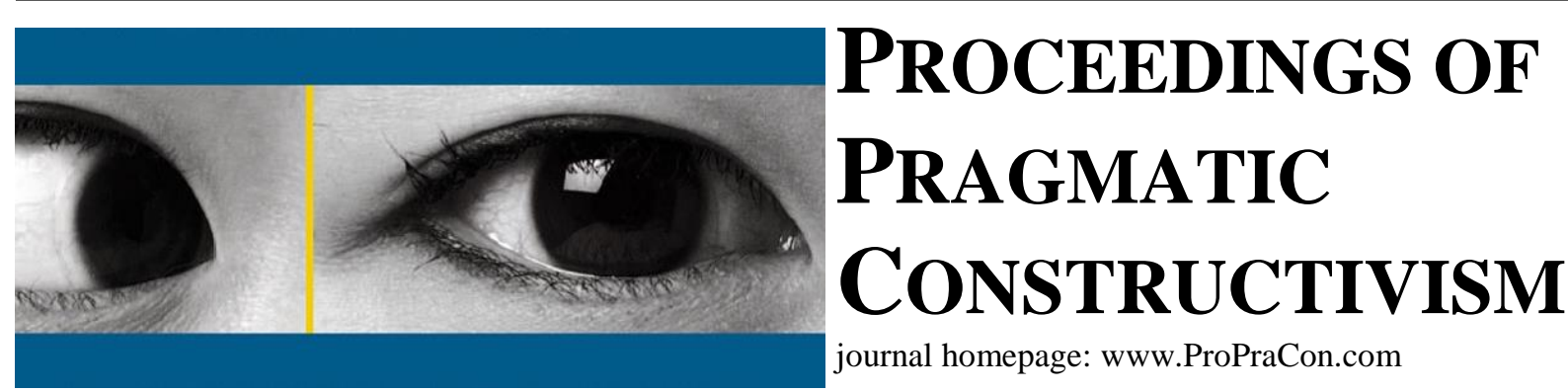

\title{
ACADEMIC-BASED NEW PRODUCT DEVELOPMENT RESEARCH PROJECTS AND MANAGEMENT ACCOUNTING PRACTICE: A PRAGMATIC CONSTRUCTIVIST APPROACH
}

\author{
Riccardo Giannetti \\ University of Pisa, Italy \\ E-mail: riccardo.giannetti@unipi.it \\ Fabio Magnacca \\ University of Pisa, Italy \\ E-mail: fabio.magnacca@ec.unipi.it \\ Giovanna Mariani \\ University of Pisa, Italy \\ E-mail: giovanna.mariani@unipi.it
}

Recent times have seen the growth of project-based temporary organisations in the process of new product development (NPD). As a result, there has been a call for more research in order to gain a better understanding of the role that accounting plays in such organisational arrangements. The aim of this paper is to heed this call by exploring the role and the use of management accounting (MA) within an academic-based NPD research project, which is a temporary organisation whose objective is to transform scientific knowledge into an exploitable innovation. Drawing on the notion of topos and the four dimensions of reality, this paper considers the project through a pragmatic constructivist lens and highlights the pivotal role of MA in this peculiar academic setting. Prior research on the role of MA in NPD in temporary organisations has not focused on this particular academic-based setting, nor on this specific anticipatory NPD research phase. The unit of analysis is neither a company nor a temporary organisation in which the participating parties are drawn from multiple companies. We use the term 'academic-based NPD research project' to refer to a research project within academia that is undertaken by a temporary organisation led by academics and researchers whose goal is to produce scientific knowledge that may be relevant to industrial stakeholders. In a development phase such as the one analysed in this paper, the level of uncertainty is extremely high, and data are usually not available. This high level of uncertainty has a direct impact on the choice and the use of MA frameworks. We claim that there are no ready-to-use MA frameworks and that these frameworks follow a process of development that goes hand in hand with the process of creating knowledge about the new product under investigation. In this context, MA techniques do not appear to be useful as they may lack numerical input. However, even if they do not provide quantitative information, MA frameworks and their underlying logic of business reality contribute to generating sense within the temporary organisation nourishing a process of reflection on business considerations and, hence, constituting a common language to talk for all the actors involved. In so doing, MA frameworks and the rationale behind them provide the actors with an awareness of the factual possibilities, which leads the research activities towards those possibilities that are economically advantageous and, therefore, relevant to potential investors. The practical implications of this 
investigation are twofold. Firstly, it helps to shed light on the reasons for and the ways in which MA is designed and used within academic-based research projects and contributes to their success. Secondly, because the role of management accountants in these kinds of projects is explored, the importance of their engagement from the very early phases is made clear. 